\title{
A comparative study between agonist and antagonist protocol for ovarian stimulation in art cycles at a rural set up in South Gujarat
}

\author{
Purnima Kishor Nadkarni ${ }^{1}$, Kishore Mohan Nadkarni ${ }^{2}$, Kanika Rajendra Kalyani ${ }^{3 *}$
}

\begin{abstract}
${ }^{1}$ Consultant Obstetrician and Gynaecologist and Infertility Specialist, Nadkarni Hospital and Test Tube Baby Centre, Killa Pardi, Vapi, Surat, Gujarat, India

${ }^{2}$ Consultant Surgeon and Infertility Specialist, Nadkarni Hospital and Test Tube Baby Centre, Killa Pardi, Vapi, Surat, Gujarat, India

${ }^{3}$ Fellow Student in Reproductive Medicine, Nadkarni Hospital and Test Tube Baby Centre, Killa Pardi, Vapi, Surat, Gujarat, India
\end{abstract}

Received: 05 March 2015

Revised: 08 March 2015

Accepted: 19 April 2015

\section{*Correspondence:}

Dr. Kanika Rajendra Kalyani,

E-mail: kanika.kalyani@gmail.com

Copyright: $($ ) the author(s), publisher and licensee Medip Academy. This is an open-access article distributed under the terms of the Creative Commons Attribution Non-Commercial License, which permits unrestricted non-commercial use, distribution, and reproduction in any medium, provided the original work is properly cited.

\begin{abstract}
Background: Modern infertility practice provides us with several protocols for controlled ovarian hyperstimulation for the ART (Assisted Reproductive Techniques) cycles. The review summarises the clinical characteristics of the protocols using Gonadotrophin Releasing Hormone $(\mathrm{GnRH})$ agonists and antagonists emphasising on the major clinical and laboratory outcomes with each protocol.

Methods: A total of 322 cases undergoing ovarian stimulation with agonist and antagonist protocols in ART cycles at a rural set up at Killa Pardi in the year 2014 were studied and their laboratory and clinical outcomes were evaluated.

Results: Antagonist group had the maximum number of oocytes retrieved and the mature M2 oocytes, maximum No. of follicles $>16 \mathrm{~mm}$ on day of HCG, maximum No. of positive pregnancy rates. Agonist group had also a good pregnancy rate with maximum Grade I embryos.

Conclusions: Taking all data together, it may be concluded that antagonists and minimal ovarian stimulation with antagonist protocols offer a new treatment regimen in ovarian stimulation that is short, safe, cost effective, well tolerated, optimizing convenience for the patient.
\end{abstract}

Keywords: GnRH agonist protocol, Antagonist protocol, Ovarian stimulation

\section{INTRODUCTION}

There are various clinical trials which demonstrate that in ICSI (Intra Cytoplasmic Sperm Injection) cycles, the combination of exogenous gonadotropin and GnRH agonists for the suppression of pituitary FSH and LH is assoaciated with higher pregnancy rates as compared to the use of gonadotropins without agonists. ${ }^{1}$ The major benefits of GnRH analogues include decreased cancellation rates through premature $\mathrm{LH}$ surge and leutinisation, ${ }^{2}$ enhancement of follicular recruitment, allowing the recovery of a larger number of oocytes, ${ }^{3}$ and the improvement in routine patient treatment schedule. A systematic overview of twenty six trials comparing different GnRH-a protocols for pituitary desensitisation in In Vitro Fertilisation demonstrated the superiority off long protocol over the short and ultrashort protocols. However, the use of long protocol is associated with an increasing requirement for gonadotropins and a longer time of ovarian stimulation. The use of GnRH agonists in the long protocol is characterised by some disadvantages for the patients; a) it has along treatment period until desensitisation occurs ${ }^{4}$ b) increased risk of ovarian hyperstimulation c) more frequent occurrence of side 
effects (e.g. Hot flushes, headache, bleeding and cyst development) during the desensitisation period. 5,6

The introduction of GnRH antagonists in ART cycles to prevent LH surge seemed to open up a new way towards a more "friendly IVF". 7 Unlike the indirect pituitary suppression induced by GnRH-a, GnRH-ant administration causes immediate and dose related inhibition of gonadotropin release by competitive occupancy of the GnRH receptors in the pituitary. The use of GnRH-ant leads to a significant reduction in the duration of ovarian stimulation. GnRH antagonists are also not associated with acute induction of gonadotropins, which may induce cyst formation. In addition, no hot flushes are observed with GnRH-ant because their use doesnot result in profound hypo-estrogenaemia observed with GnRH-a. Finally, a reduced incidence of OHSS is seen with GnRH-ant. In our experience, the avoidance of acute stimulation of endogenous gonadotropins, the short duration of treatment, and the ability to inhibit directly the premature LH surge made GnRH-ant one of the most appropriate regimen for ovarian stimulation and for embryo transfer.

\section{GnRH antagonists - Fixed versus flexible regimen}

Defining the most appropriate time to start cetrorelix administration has been the subject of several studies. The most common type of treatment called fixed protocol consists of giving GnRH-ant 5 days after the stimulation with gonadotrophins. However in order to reduce the number of antagonist injections and the duration of stimulation, the flexible protocol was introduced. It consists in administering GnRH antagonist when the follicles reach a size of $>14 \mathrm{~mm} .{ }^{8,9}$

\section{METHODS}

A total of 322 patients in whom ICSI was indicated and who fulfilled the selection criteria were studied. These were the patients who underwent controlled ovarian hyperstimulation at Nadkarni Hospital and Test Tube Baby Centre, Killa Pardi, a rural centre in South Gujarat in the year 2014. In 116 patients, FSH was given from day 2 or day 3 of menses until the day of HCG. On day 8, when the follicles were more than $14 \mathrm{~mm}$ size, cetrorelix $0.25 \mathrm{mg}$ was started and given for four days or until the day of HCG administration. This was the antagonist protocol. In 123 patients, pretreatment with triptorelin was started on day 21 of the preceding cycle (mid luteal phase) or from day 2 of menses until HCG injection and in some on only day 2, 3 and 4 of menses. This was decided according to the patient's age, body mass index, antral follicle count, antimullerian horrmone levels and day 2 serum FSH levels.

The daily dose of triptorelin was $0.1 \mathrm{mg}$ subcutaneous in the upper leg or abdomen. Ovarian stimulation started after 2 weeks of treatment when downregulation was established (Serum estradiol $<50 \mathrm{pg} / \mathrm{ml}, \mathrm{LH}<5 \mathrm{ng} / \mathrm{ml}$ ). If downregulation was not achieved after 21 days, subject was discontinued.

For subjects in either regimens, ovarian stimulation was started and adjusted according to the age, BMI, AMH, FSH, antral follicle count for the first 5 days. From day 6 onwards, the daily dose was adjusted and individualised per subject based on the follicular growth as observed by ultrasonography. On the day of HCG administration, no treatment with FSH was applied.

HCG was administered when atleast 3 follicles of $>17$ $\mathrm{mm}$ were measured by USG. About 34 hours after HCG administration, oocyte retrieval was performed with the help of transvaginal ultrasound followed by ICSI. 2 embryos were transferred on day 2 followed by a sequential blastocyst transfer on day 5. Progesterone was given for luteal phase support according to the Centre's routine practice.

\section{RESULTS}

Mean age of patients who were given antagonist protocol was $31.06 \pm 4.62$ years and in the agonist protocol group, it was $32.36 \pm 5.66$ years. The women who needed minimal ovarian stimulation with antagonist protocol were relatively younger with an average age of $28.43 \pm$ 4.54 years. And similarly the serum FSH values were higher $(15.41 \pm 70.57,15.12 \pm 61.36)$ in the antagonist and agonist group respectively but were within the normal range i.e. $6.28 \pm 3.60$ in patients who required minimal ovarian stimulation with antagonist protocol. Luteinising hormone levels were normal in patients in the GnRH-ant group but were higher in the agonist group $(15.18 \pm 76.32)$ which is why they were given downregulation. Almost all patients had a similar antral follicle count which was 8-10 and a normal value of AMH. Various causes of infertility were evenly distributed amongst all patients like endometriosis, tubal factor, unexplained infertility, etc.

During ovarian stimulation with all these protocols, it was surprising to know that number of follicles $>16 \mathrm{~mm}$ were seen maximum in the antagonist group i.e. $10.01 \pm$ $4.45 \mathrm{~mm}$ followed by MOS-ant group $(9.37 \pm 3.15 \mathrm{~mm})$ and the agonist group $(8.92 \pm 4.07 \mathrm{~mm})$. Endometrial thickness on the day of embryo transfer was good in all the three protocols which were between 10 to $11 \mathrm{~mm}$ in all the three groups. This proved that even antagonist protocols gave good Endometrium for implantation.

To our surprise, the number of oocytes retrieved were maximum in the antagonist group i.e. $14.19 \pm 6.84$ followed by the agonist group $(10.52 \pm 5.65)$ and the MOS-ant group $(13.23 \pm 11.13)$. GnRH-ant group had the maximum number of mature M2 oocytes $(10.36 \pm 4.90)$, maximum number of fertilised oocytes $(9.05 \pm 18.04)$ and also the maximum number of embryos cleaved $(9.13 \pm$ 18.10). But on the contrary the agonist group gave a good quality of embryos since we obtained maximum grade I 
embryos [68 (21.11\%)] in the agonist group. The GnRHant and MOS-ant group also had a comparable number of Grade I embryos 56 (17.39\%) and 44 (13.66\%) respectively). Blastocyst formation rate was same in all the three protocols. Thus antagonist protocols had a similar blastocyst formation rate as in the agonist protocol which resulted in better implantation again. Overall Beta HCG positive rate was $55.5 \%$ out of which maximum i.e. $70(21.73 \%)$ belonged to the antagonist group followed by the agonist group [55 (17.08\%)] and the MOS-ant group [54 (16.77\%)].

Thus, the antagonist protocol was in no way inferior to the agonist group. Infact out of 116 patients in whom the GnRH-ant protocol was followed, 70 patients had their Beta HCG positive giving a rate of $60.34 \%$. Since MOS- ant was given to younger women with better ovarian reserve, out of 83 women undergoing stimulation according to this regimen, 54 became pregnant giving the maximum rate of $65.06 \%$. Agonist group had a beta HCG positive rate of around $45 \%$.

Table 1: Distribution of protocols.

\begin{tabular}{|lll|}
\hline Protocol & $\begin{array}{l}\text { No. of } \\
\text { patients }\end{array}$ & Percent \\
\hline Antagonist & 116 & 36.02 \\
\hline Agonist & 123 & 38.20 \\
\hline MOS-antagonist & 83 & 25.78 \\
\hline Total & 322 & 100.0 \\
\hline
\end{tabular}

Table 2: Baseline characteristics.

\begin{tabular}{|llllll|}
\hline Parameters & Antagonist & Agonist & MOS-antagonist & F-value & P value \\
\hline Age (years) & $31.06 \pm 4.62$ & $32.36 \pm 5.66$ & $28.43 \pm 4.54$ & 29.20 & $0.000 \mathrm{~S}, \mathrm{P}<0.05$ \\
\hline Sr. FSH & $15.41 \pm 70.57$ & $15.12 \pm 61.36$ & $6.28 \pm 3.60$ & 0.83 & $0.47 \mathrm{NS}, \mathrm{P}>0.05$ \\
\hline Sr. LH & $6.65 \pm 5.68$ & $15.18 \pm 76.32$ & $6.43 \pm 3.57$ & 0.90 & $0.43 \mathrm{NS}, \mathrm{P}>0.05$ \\
\hline Sr. E2 & $59.68 \pm 60.08$ & $57.14 \pm 76.18$ & $89.98 \pm 157.47$ & 2.80 & $0.041 \mathrm{~S}, \mathrm{P}<0.05$ \\
\hline TSH & $22.22 \pm 26.86$ & $15.88 \pm 11.13$ & $19.22 \pm 12.59$ & 2.51 & $0.059 \mathrm{NS}, \mathrm{P}>0.05$ \\
\hline AMH & $3.24 \pm 3.83$ & $2.72 \pm 2.94$ & $2.71 \pm 1.89$ & 1.17 & $0.31 \mathrm{NS}, \mathrm{P}>0.05$ \\
\hline No. of follicles & $10.01 \pm 4.45$ & $8.92 \pm 4.07$ & $9.37 \pm 3.15$ & 1.29 & $0.29 \mathrm{NS}, \mathrm{P}>0.05$ \\
\hline Cause of infertility & $7.19 \pm 4.00$ & $8.32 \pm 18.25$ & $6.53 \pm 3.85$ & 1.22 & $0.30 \mathrm{NS}, \mathrm{P}>0.05$ \\
\hline Endometriosis & $11.45 \pm 2.36$ & $11.14 \pm 2.21$ & $10.69 \pm 2.03$ & 1.52 & $0.36 \mathrm{NS}, \mathrm{P}>0.05$ \\
\hline Tubal factor & $4(1.24 \%)$ & $3(0.93 \%)$ & $2(0.62 \%)$ & 0.00 & $1.00 \mathrm{NS}, \mathrm{P}>0.05$ \\
\hline Male factor & $40(12.42 \%)$ & $34(10.56 \%)$ & $20(6.21 \%)$ & 2.36 & $0.30 \mathrm{NS}, \mathrm{P}>0.05$ \\
\hline PCO & $13(4.04 \%)$ & $0(0 \%)$ & $8(2.48 \%)$ & 4.08 & $0.12 \mathrm{NS}, \mathrm{P}>0.05$ \\
\hline Unexplained & $59(18.32 \%)$ & $82(25.47 \%)$ & $53(16.46 \%)$ & 2.82 & $0.42 \mathrm{NS}, \mathrm{P}>0.05$ \\
\hline
\end{tabular}

Table 3: During stimulation.

\begin{tabular}{|llllll|} 
& Antagonist & Agonist & MOS-antagonist & F-value & P value \\
\hline No. of follicles $>16 \mathrm{~mm}$ & $10.01 \pm 4.45$ & $8.92 \pm 4.07$ & $9.37 \pm 3.15$ & 7.13 & $0.000 \mathrm{~S}, \mathrm{P}<0.05$ \\
\hline Endometrial thickness on the day of ET & $11.45 \pm 2.35$ & $11.14 \pm 2.21$ & $10.69 \pm 2.03$ & 16.55 & $0.000 \mathrm{~S}, \mathrm{P}<0.05$ \\
\hline
\end{tabular}

Table 4: Laboratory and clinical outcomes.

\begin{tabular}{|llllll|} 
& Antagonist & Agonist & MOS-antagonist & F-value & P value \\
\hline No. of oocytes retrieved & $14.19 \pm 6.84$ & $10.52 \pm 5.65$ & $13.23 \pm 11.13$ & 5.87 & $0.003 \mathrm{~S}, \mathrm{P}<0.05$ \\
\hline No. of mature (M2) oocytes & $10.36 \pm 4.90$ & $8.21 \pm 3.84$ & $8.32 \pm 4.80$ & 7.27 & $0.001 \mathrm{~S}, \mathrm{P}<0.05$ \\
\hline No. of fertilized oocytes & $9.05 \pm 18.04$ & $2.87 \pm 0.29$ & $6.12 \pm 3.91$ & 2.25 & $0.107 \mathrm{NS}, \mathrm{P}>0.05$ \\
\hline No. of embryos cleaved & $9.13 \pm 18.10$ & $5.93 \pm 2.82$ & $6.08 \pm 3.83$ & 2.42 & $0.090 \mathrm{NS}, \mathrm{P}>0.05$ \\
\hline No. of embryos formed & & & & \\
\hline Grade 1 & $56(17.39 \%)$ & $68(21.11 \%)$ & $44(13.66 \%)$ & \multirow{2}{*}{1.02} & $0.59 \mathrm{NS}, \mathrm{P}>0.05$ \\
\hline Grade 2 & $7(2.17 \%)$ & $6(1.86 \%)$ & $7(2.17 \%)$ & 0.215 & $0.807 \mathrm{NS}, \mathrm{P}>0.05$ \\
\hline No. Of blastocysts formed & $3.01 \pm 0.70$ & $3.03 \pm 0.67$ & $3.08 \pm 0.51$ & 4.37 & $0.112 \mathrm{NS}, \mathrm{P}>0.05$ \\
\hline Beta HCG positive & $70(21.73 \%)$ & $55(17.08 \%)$ & $54(16.77 \%)$ & & \\
\hline
\end{tabular}




\section{DISCUSSION}

The enhancement of sensitivity for patients in response to controlled ovarian hyperstimulation is a pivotal factor associated with successful clinical pregnancy during the IVF-ET treatment. ${ }^{10}$ Overall our study demonstrates a comparable and in fact better efficacy and safety of GnRH-ant protocol. Previous studies have shown a lower clinical and on-going pregnancy rates for the GnRH-ant protocols but these studies show some confounding variables from a methodological point of view. Mostly the data were pooled from patients with previously failed IVF attempts i.e. patients with advanced age and with a higher number of previously unfavourable cycles, thereby carrying a possible risk of introducing confounding factors. Our findings of higher implantation rate in the antagonist group were acceded by another study by Qiaohong Lai et al. who also found a higher implantation rate and clinical pregnancy rate. Their study also stated that GnRH antagonist protocol could be more efficient for improving the outcome of pregnancy in those patients with a history of multiple failures for the IVF-ET treatment. Data from some randomized clinical trials revealed that the antagonist protocol retrieves less number of oocytes along with lower pregnancy rates than the agonist long protocol. ${ }^{11,12}$ More recently, some metaanalysis based studies failed to suggest a significant difference in terms of pregnancy outcomes between these two protocols. In our study also, the number of oocytes retrieved were maximum in the antagonist group i.e. $14.19 \pm 6.84$ followed by the agonist group (10.52 \pm $5.65)$ and the MOS-ant group $(13.23 \pm 11.13)$. Given the fact that our dataset only contains limited number of patients, future studies with more subjects and stimulation cycles would be necessary to further confirm those observations. In the study by Qiaohong Lai et al., embryo quality was better with the antagonist protocol whereas in our study maximum grade I embryos were seen in the subjects who underwent ovarian stimulation with the agonist protocol. GnRH agonists have been widely used in controlled ovarian hyperstimulation during the IVF-ET treatment. In contrast, the clinical application time for GnRH antagonists is relatively short, and their impact on the outcome of IVF-ET treatment, however, is yet to be fully elucidated. The studies in our dataset demonstrated feasible advantage for the antagonist protocol over the agonist long protocol in terms of implantation rate and pregnancy rate, particularly in those patients with multiple failures for the agonist protocol. However, additional studies with more subjects and stimulation cycles would be necessary to further confirm these data. It would be also necessary to optimize the protocol and to conduct studies for better understanding its effect on endometrium.

\section{CONCLUSION}

Taking all data together, it may be concluded that the GnRH-ant protocol and MOS-ant protocol offer a new treatment regimen in ovarian stimulation that is short, safe, cost effective, well tolerated, optimising convenience for the patient.

Funding: No funding sources

Conflict of interest: None declared

Ethical approval: The study was approved by the institutional ethics committee

\section{REFERENCES}

1. Depalo R, Jayakrishan K, Garruti G, Totaro I, Panzarino M, Giorgino F, et al. GnRH agonist versus GnRH antagonist in invitro fertilization and embryo transfer (IVF/ET). Reprod Biol Endocrinol. 2012;10:26.

2. Caspi E, Ron-El R, Golan, Nachum H, Herman A, Soffer Y, et al. Results of in vitro fertilisation and embryo transfer by combined long acting gonadotropin releasing hormone analog D-Trp-6luteinizing hormone releasing hormone and gonadotropins. Fertil Steril. 1989;51(1):95-9.

3. Liu HC, Lai YM, Davis O, Berkeley AS, Graf M, Grifo J, et al. Improved pregnancy outcome with gonadotropin releasing hormone agonist ( $\mathrm{GnRH}-\mathrm{a})$ stimulation is due to the improvement in oocyte quantity rather than quality. J Assist Reprod Genet. 1992;9(4):338-44.

4. Conn PM, Staley D, Harris C, Andrews WV, Gorospe WC, McArdle CA. Mechanism of action of gonadotropin releasing hormone. Annu Rev Physiol. 1986;48:495-513.

5. Rizk B, Smith J. Ovarian hyperstimulation syndrome after superovulation using GnRH agonists for IVF and related procedures. Hum Reprod. 1992;7(3):3207 .

6. Ben-Rafael Z, Lipitz S, Bider D, Mashiach S. Ovarian hyporesponsiveness in combined gonadotropin-releasing hormone agonist and menotropin therapy is associated with low serum follicle stimulating hormone levels. Fertil Steril. 1991;55(2):272-5.

7. Ron-El R, Herman A, Golan A, Raziel A, Soffer Y, Caspi E. Follicle cyst formation following long acting gonadotropin releasing hormone analog administration. Fertil Steril. 1989;52(6):1063-6.

8. Olivennes F, Frydman R. Friendly IVF: the way of the future. Human Reprod. 1998;13(5):1121-4.

9. Ludwig M, Katalinic A, Banz C, Schroder AK, Lonong M, Weiss JM, et al. Tailoring the GnRH antagonist cetrorelix acetate to individual patients' needs in ovarian stimulation for IVF and results of the perspective. Fertil Steril. 2000;73(2):314-20.

10. Prapas Y, Pettousis S, Dagklis T, Panagiotidis Y, Papatheodorou A, Assunta I, et al. GnRH antagonist versus long GnRH agonist protocol in poor IVF responders: a randomized clinical trial. Eur J Obstet Gynecol Reprod Biol. 2013;166:43-6.

11. Kim CH, You RM, Kang HJ, Ahn JW, Jeon I, Lee JW, et al. GnRH antagonist multiple dose protocol with oral contraceptive pill pretreatment in poor 
responders undergoing IVF/ICSI. Clin Exp Reprod Med. 2011;38:228-33.

12. Andersen AN, Witjes H, Gordon K, Mannaerts B. Predictive factors of ovarian response and clinical outcome after IVF/ICSI following a $\mathrm{rFSH} / \mathrm{GnRH}$ antagonist protocol with or without oral contraceptive pre-treatment. Hum Reprod. 2011;26:3413-23.

DOI: $10.18203 / 2320-1770.1 j \mathrm{rcog} 20150062$

Cite this article as: Nadkarni PK, Nadkarni KM,

Kalyani KR. A comparative study between agonist and antagonist protocol for ovarian stimulation in art cycles at a rural set up in South Gujarat. Int J Reprod Contracept Obstet Gynecol 2015;4:617-21. 